\title{
Characterization of Small GTPases Cdc42 and Rac and the Relationship Between Cdc42 and Actin Cytoskeleton in Vegetative and Ectomycorrhizal Hyphae of Suillus bovinus
}

\author{
Markus Gorfer, Mika T. Tarkka, Mubashir Hanif, Alejandro G. Pardo, Erja Laitiainen, and \\ Marjatta Raudaskoski \\ Department of Biosciences, Division of Plant Physiology, P.O. Box 56, 00014 University of Helsinki, Finland \\ Accepted 19 October 2000.
}

This work reports the isolation and molecular characterization of $C D C 42$ and $R A C 1$ cDNAs from the ectomycorrhiza forming filamentous homobasidiomycete Suillus bovinus. Previously, no $R A C$ gene was described from filamentous fungi and no $C D C 42$ gene was described from homobasidiomycetes. Southern hybridization with $S b C D C 42$ and SbRAC1 cDNAs indicated that the $S$. bovinus genome contains only one $C D C 42$ and one $R A C 1$ gene. The predicted amino acid sequence of SbRac1p is $77 \%$ identical with the Rac1B protein of chick, whereas SbCdc42p is most identical with Schizosaccharomyces pombe Cdc42p, showing $88 \%$ identity. In the predicted amino acid sequences of SbRac1p and SbCdc42p, the five guanine nucleotide binding regions, switch I and II, and the effector domain are highly identical to those known in other small GTPases. These domain structures suggest that in $S$. bovinus, SbRac1p and SbCdc42p function as molecular switches regulating the organization of actin cytoskeleton, similar to yeasts and mammals. $S b R A C 1$ and $S b C D C 42$ were expressed in vegetative and ectomycorrhizal hyphae, and SbCdc42p was detected in ectomycorrhiza-forming hyphae if growth and differentiation of the symbiotic hyphae took place. Cdc42p and actin were localized at the tips of $S$. bovinus vegetative hyphae. Similar to yeast, in filamentous fungi Cdc42p may be necessary to maintain the actin cytoskeleton at hyphal tips, making the polarized growth of the hyphae possible. In developing ectomycorrhiza, Cdc42p and actin were visualized in association with plasma membrane in swollen cells typical to the symbiotic hyphae. The role of $\mathrm{Cdc42p}$ and actin in regulation of the growth pattern and morphogenesis of ectomycorrhizal hyphae is discussed.

Corresponding author: M. Raudaskoski;

E-mail: marjatta.raudaskoski@helsinki.fi

Genbank accession numbers for nucleotide and amino acid sequences: SbCdc42, AF234180; and SbRac1, AF235004.

A. G. Pardo is a visiting researcher from Programa de Investigación en Interacciones Biológicas, Centro de Estudios e Investigaciones, Universidad Nacional de Quilmes, Roque Sáenz Peña 180, (B1876BXD) Bernal, Provincia de Buenos Aires, Argentina, and a member of the Scientific Research Career of CONICET (Argentina).
Additional keywords: signal transduction, symbiosis.

When the ectomycorrhizal fungus Suillus bovinus forms mycorrhizae with Pinus sylvestris roots, the polarized hyphae are replaced by branched swollen hyphal compartments. These morphological differences between nonsymbiotic and symbiotic hyphae are well known (Kottke and Oberwinkler 1986; Timonen et al. 1993), but factors regulating the change in the growth pattern are not known. In eukaryotic organisms the reorganization of cell structure is based on signal-mediated changes in actin network and in the activities of associated proteins. The proteins involved in the control of actin cytoskeleton reorganization are best known in mammalian cells and in the budding yeast Saccharomyces cerevisiae, where Rho-like GTPases act as key regulators in signaling pathways, linking extracellular signals and intracellular stimuli to the assembly and organization of actin cytoskeleton (Schmidt and Hall 1998). In order to mediate signaling to their downstream effector proteins, the small GTPases cycle between an inactive and an active GTP-bound form (Nuoffer and Balch 1994). In filamentous fungi, only a few studies have identified components associated with signal transduction pathways regulating hyphal morphogenesis during saprotrophic growth (Hicks et al. 1997; Rosen et al. 1999), pathogenesis, or sexual reproduction (Bölker 1998). None of these investigations focus on ectomycorrhiza-forming filamentous fungi such as S. bovinus or the involvement of small GTPases in regulating the hyphal morphogenesis at ectomycorrhiza formation.

Here we report the isolation of two small GTPases of the Rho subfamily, $C D C 42$ and $R A C 1$, from an ectomycorrhizaforming fungus. $C D C 42$ was first isolated in $S$. cerevisiae by complementation of a temperature-sensitive $c d c 42$ mutant (Johnson and Pringle 1990). The mutation in CDC42 inhibited bud formation, and fluorescence microscopy with rhodamineconjugated phalloidin showed that the mutation caused disruption of the actin cytoskeleton. These features indicated that the function of Cdc42p is involved with the organization of actin cytoskeleton necessary for polarized cell growth, which was confirmed profoundly in later studies (Johnson 1999). In mammalian cells, Cdc42p, after activation by a specific extracellular signal bound to a G-protein coupled receptor, directs 
the organization of actin to form filopodia and peripheral actin microspikes (Schmidt and Hall 1998). The small GTPase Rac is absent from $S$. cerevisiae, but is apparent in mammalian cells, where it controls the actin polymerization necessary for lamellipodia formation. In phagocytic cells, Rac protein also is known as part of the multienzyme complex NADPH oxidase, which produces superoxide in response to invading microorganisms. In plant cells, Rac protein was shown to colocalize with microtubules (Couchy et al. 1998) and regulate cell death (Kawasaki et al. 1999).

We analyzed the expression of the cloned CDC42 and $R A C l$ genes in vegetative hyphae of $S$. bovinus and in the $S$. bovinus-P. sylvestris ectomycorrhiza by Northern hybridization. The distribution of $\mathrm{Cdc} 42 \mathrm{p}$ in the fungal hyphae and ectomycorrhiza was further confirmed by Western blotting and indirect immunofluorescence (IIF) microscopy.

\section{RESULTS}

\section{Cloning of $S b R A C 1$ and $S b C D C 42$ genes.}

Polymerase chain reaction (PCR) with genomic DNA from the ectomycorrhizal fungus $S$. bovinus and the ras superfamily primers led to isolation and subsequent cloning of 99 PCR fragments. The primers were chosen on the basis of conserved regions of the 5 and $3^{\prime}$ ends in the nucleotide binding regions of small GTPase-encoding genes. Restriction fragment length polymorphism (RFLP) analysis of the PCR fragments separated them into 11 different groups, and sequencing of representatives from each group identified six different ras-family members, out of which two ras and three rab homologs were identified. Fourteen clones showing identical RFLP patterns contained a 318 -bp insert with $92 \%$ identity at the amino acid level to the protist Dictyostelium discoideum Rac1A. The fragment was used to screen the existing cDNA library prepared from $S$. bovinus vegetative hyphae, and one cDNA clone, SbRAC1, was isolated and sequenced. The SbRAC1 cDNA consisted of $801 \mathrm{bp}$, out of which $123 \mathrm{bp}$ belonged to $5^{\prime}$ and 85 bp to $3^{\prime}$ untranslated region (UTR) (Fig. 1). The predicted amino acid sequence consisted of 194 residues showing $77 \%$ identity with the Rac1B protein and $76 \%$ identity with Yarrowia lipolytica Rac1p, but only $72 \%$ identity with the full-length $D$. discoideum Rac1A protein. No $R A C$ was identified previously from filamentous fungi.

No genomic CDC42 fragment could be amplified with the ras superfamily primers. The PCR with novel primers specific for $C D C 42$ led to the isolation of a 640-bp fragment of the $S b C D C 42$ gene, which was sequenced. In the $S b C D C 42$ gene fragment, both of the ras superfamily primer binding sites were interrupted by introns. The predicted amino acid sequence of the partial $S b C D C 42$ gene showed $88 \%$ identity to Cdc42p of Schizosaccharomyces pombe. The screening of the $S$. bovinus cDNA library with the PCR fragment led to identification of 12 positive clones. Four of the clones were isolated on the basis of a small variety of cDNA sizes in restriction enzyme analysis and sequenced. All clones contained an open reading frame (ORF) of $579 \mathrm{bp}$, which encodes 193 amino acid residues showing $88 \%$ identity to $S$. pombe Cdc42p, $82 \%$ identity to Aspergillus nidulans modA, $80 \%$ identity to Homo sapiens $\mathrm{Cdc} 42$, and $73 \%$ identity to Colletotrichum gloeosporioides Cdc42p. Two of the $S b C D C 42$ cDNAs were shorter from their $5^{\prime}$ UTRs (Fig. 1), perhaps as a result of in- complete first-strand cDNA synthesis. In the $5^{\prime}$ UTR of three of the four isolated cDNAs, an 18-bp insertion was detected. The insertion included a conserved 3 '-intron-end sequence and a branching-pointlike sequence. The significance of this 18-bp $5^{\prime}$-UTR sequence can be clarified only when the corresponding $\operatorname{SbCDC42}$ gene is isolated. The 3' UTR of cDNAs was either 138, 311 (two clones) or $471 \mathrm{bp}$. In the second-longest 3' UTR, a signature for polyadenylation located $23 \mathrm{bp}$ upstream from the poly(A)-tail was identified, whereas in the longest 3' UTR, a putative polyadenylation signal $37 \mathrm{bp}$ from poly-(A)-tail was detected (Fig. 1).

\section{Comparison of SbCdc42p and SbRac1p predicted amino acid sequences.}

The predicted amino acid sequences of SbRACl and $S b C D C 42$ genes are $63 \%$ identical. The main difference occurs in and close to the "insert region," from amino acid (aa) 123 to 135 , which is typical to the Rho family, but absent from Ras family proteins (Nuoffer and Balch 1994). Analysis of the amino acid sequences predicts that SbRaclp and SbCdc42p

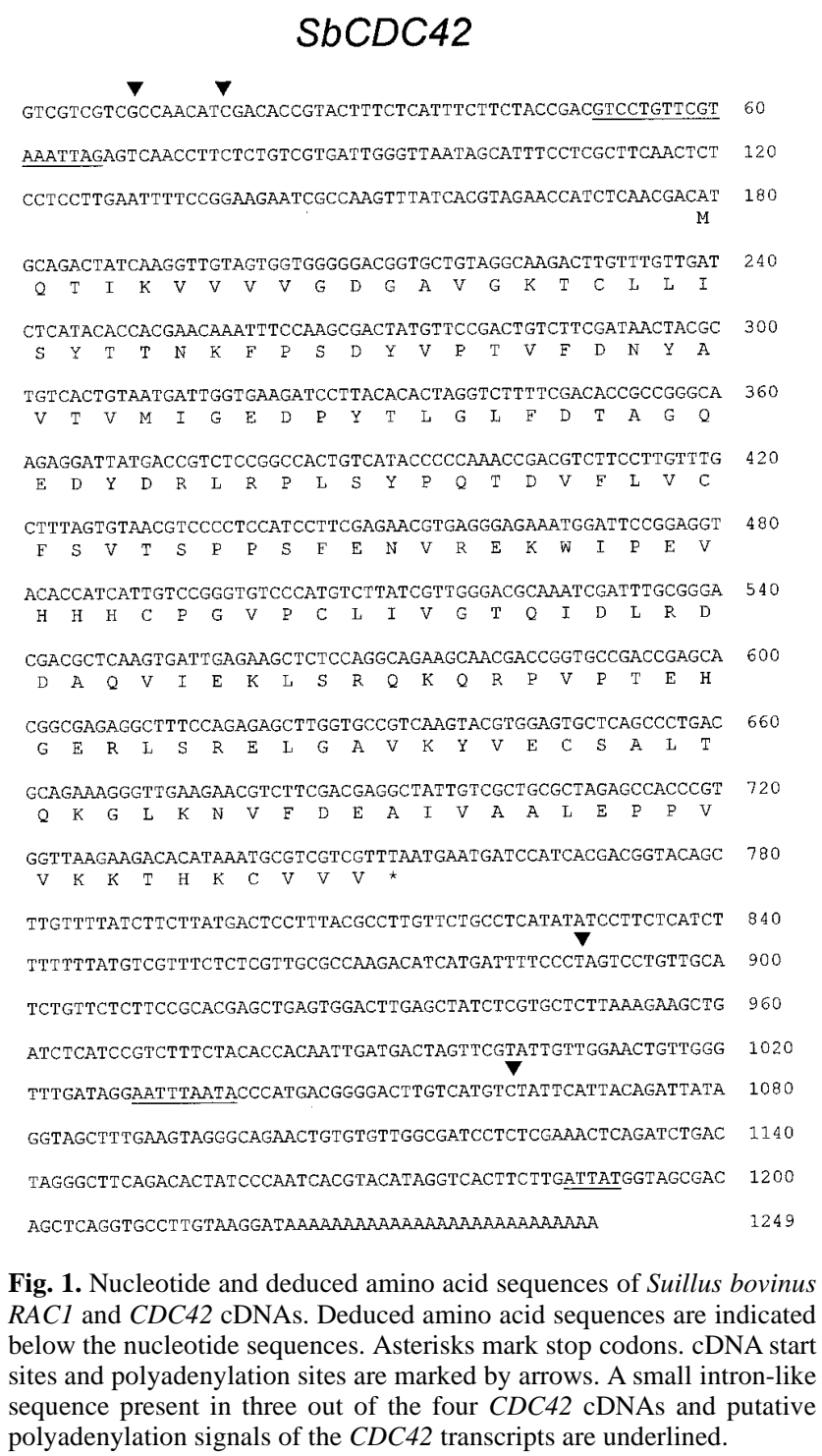

Fig. 1. Nucleotide and deduced amino acid sequences of Suillus bovinus $R A C 1$ and $C D C 42$ cDNAs. Deduced amino acid sequences are indicated below the nucleotide sequences. Asterisks mark stop codons. cDNA start sites and polyadenylation sites are marked by arrows. A small intron-like sequence present in three out of the four CDC42 cDNAs and putative polyadenylation signals of the $C D C 42$ transcripts are underlined. 
contain all five functional domains associated with binding and hydrolysis of guanine nucleotides (Fig. 2). These domains are almost identical in SbCdc42p and SbRaclp and highly homologous to those in mammals and yeasts. The deduced amino acid sequences of SbCdc42p and SbRac1p also contain a carboxyl-terminal consensus sequence, CAAX (C, cysteine; $\mathrm{A}$, any aliphatic amino acid; and $\mathrm{X}$, any amino acid), which leads to posttranslational modification and prenylation of the $\mathrm{Rac}$ and $\mathrm{Cdc} 42$ proteins and localization of the proteins to the inner plasma membrane (Omer and Gibbs 1994). SbRac1p is three amino acids longer than SbCdc42p. The three additional amino acids of SbRac1p are located before the CAAX prenylation signal (Fig. 2). In the predicted SbCdc42p amino acid sequence, aa 138 is proline rather than threonine and aa 186 is histidine (His) rather than lysine or arginine (Fig. 2), compared with most other $\mathrm{Cdc} 42$ proteins. Whether these differences are of any significance to the function of SbCdc $42 p$ remains to be clarified. Similarly, the Cdc $42 p$ of filamentous fungus $C$. gloeosporioides has a His at aa 186 in its polybasic carboxyl terminal (Fig. 3).

Molecular phylogenetic analysis was performed to examine the evolutionary relationships of predicted $S$. bovinus $\mathrm{Cdc} 42$ and Rac amino acid sequences with their eukaryotic homologs. A phylogenetic tree (Fig. 4) of full-length amino acid sequences was constructed by the neighbor-joining method in the PHYLIP program (Phylogeny Interference Package; Felsenstein 1989). All fungal full-length Cdc42 or Rac proteins available in GenBank, EMBL, and SwissProt were included in the analysis beside representative members of animal $\mathrm{Cdc} 42$ and Rac proteins. Plant Rac protein (Arabidopsis thaliana Rac1) was used as an outgroup because plant Rac proteins showed low similarity with most other Rac proteins. The obtained tree clearly shows that the described small GTPases of $S$. bovinus represent members of the Cdc42 and Rac families.

\section{Southern and Northern hybridization.}

Southern hybridization of BamHI-, EcoRI-, and SalIdigested genomic DNA hybridized with $S b C D C 42$ and SbRAC1 cDNAs suggested a single copy of CDC42 and
$R A C 1$ genes in the S. bovinus genome (Fig. 5A and B). Southern hybridization of SalI-digested DNA with RACl cDNA led to the detection of two hybridization fragments (Fig. 5B), which agrees with the occurrence of a SalI site in the SbRACI cDNA.

Total RNA isolated from $P$. sylvestris short roots, pooled ectomycorrhizal short roots of different developmental stages of $P$. sylvestris-S. bovinus ectomycorrhiza (very young, dichotomously branched, and coralloid ectomycorrhizas), and $S$. bovinus nonsymbiotic hyphae were analyzed by Northern hybridization. Probing the Northern blots with $S b C D C 42$ and SbRAC1 cDNAs showed that CDC42 and RAC1 were expressed in vegetative hyphae and in hyphae, forming ectomycorrhiza with $P$. sylvestris short roots. No signal for $C D C 42$ or $R A C 1$ expression was detected in Northern hybridization of total RNA isolated from $P$. sylvestris short roots. The hybridization patterns of $S b C D C 42$ and $S b R A C 1$ cDNAs were comparable to the one obtained by SbACT1 cDNA (Fig. 5E). The

Hsapiens
Mmusculus
Ggallus
Dmelanogaster
Celegans
Anidulans
Edermatitidis
Calbicans
Scerevisiae
Sbovinus
Spombe
Cgloesporioides

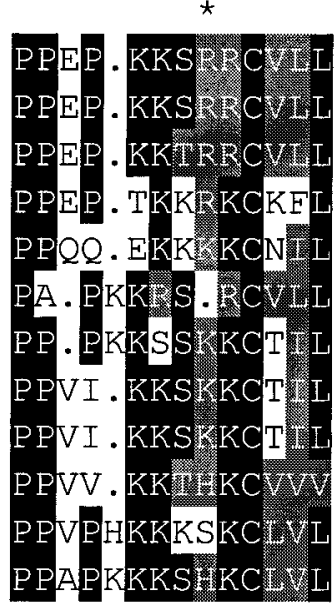

Fig. 3. Multiple alignment of the last 14 amino acids of the Cdc42 amino acid sequences. Amino acid 186 is marked by an asterisk. For accession numbers see Figure 4 .

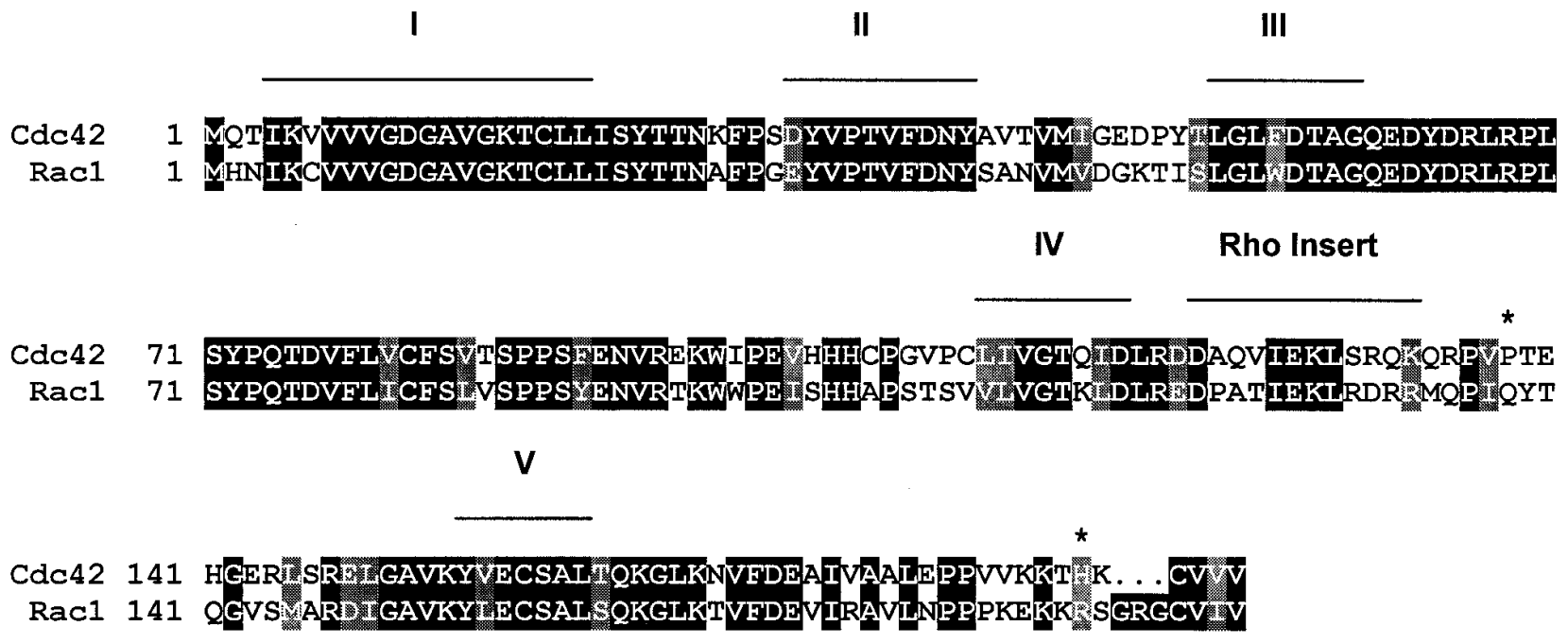

Fig. 2. Sequence alignment of Cdc42p and Rac1p from Suillus bovinus. Lines above the sequences indicate amino acids comprising the five GTP binding-hydrolysis domains and Rho insert region. Asterisks mark Cdc42p amino acids P138 and H186. 
levels of $S b C D C 42, S b R A C 1$, and $S b A C T 1$ transcripts were equal in the symbiotic and nonsymbiotic hyphae, whereas the level of $S b C D C 42$ transcripts was clearly higher than those of SbRAC1.

\section{Western blotting.}

Expression of SbCdc42p also was studied by Western blotting with a polyclonal antibody raised against yeast $\mathrm{Cdc} 42 \mathrm{p}$. The antibody recognized in the crude hyphal extracts one band with a molecular weight close to $21 \mathrm{kDa}$ (Fig. 6A and C, lane $\mathrm{S}$ ), which is at the same position as yeast Cdc42p (Fig. 6C, lane Y). For Western blotting, protein extracts were prepared

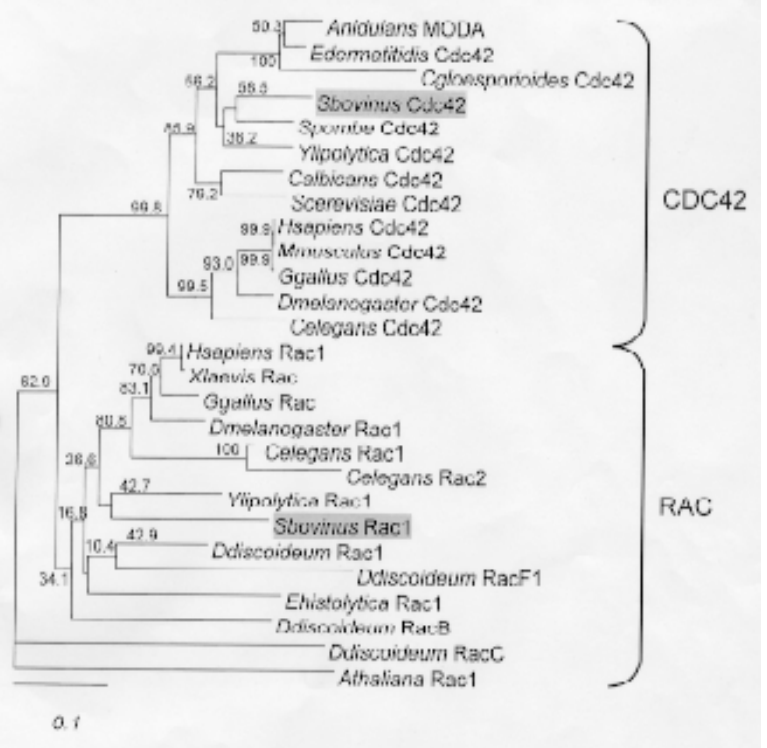

Fig. 4. Molecular phylogenetic analysis performed on the basis of amino acid differences between predicted full length $\mathrm{Cdc} 42$ and Rac amino acid sequences. A plant Rac (Arabidopsis thaliana Rac1) was included as an outgroup. The tree was inferred by the neighbor-joining method. Branch lengths are proportional to evolutionary distances. Scale bar indicates an evolutionary distance of 0.1 amino acid substitutions per position in the sequence. A thousand bootstraps were used. Numbers show the percentage of replicates that contained the presented branches. The low bootstrap values inside the group of Rac sequences do not indicate a high significance of different branches, but the presented branching order was obtained with Fitch-Margoliash and maximum parsimony analyses (not shown). GenBank, EMBL, and SwissProt accession nos.: Athaliana (Arabidopsis thaliana) rac1, AF115466; Ddiscoideum (Dictyostelium discoideum) racC, P34149; Celegans (Caenorhabditis elegans) cdc42, U23452.1; Dmelanogaster (Drosophila melanogaster) cdc42, P40793; Ggallus (Gallus gallus) cdc42, Q90694; Mmusculus (Mus musculus) cdc42, NP_033991; Hsapiens (Homo sapiens) cdc42, AAA52494; Scerevisiae (Saccharomyces cerevisiae) cdc42, X51906; Calbicans (Candida albicans) cdc42, O14426; Ylipolytica (Yarrowia lipolytica) cdc42, AF209750; Spombe (Schizosaccharomyces pombe) cdc42, Q01112; Sbovinus (Suillus bovinus) cdc42, AF234180; Cgloesporioides (Colletotrichum gloesporioides) cdc42, U70875; Edermatitidis (Exophiala dermatitidis) cdc42, AF162788; Anidulans MODA (Aspergillus nidulans modA), AF217198; Ddiscoideum (Dictyostelium discoideum) racB, P34148; Ehistolytica (Entamoeba histolytica) racA, Q24814; Ddiscoideum (Dictyostelium discoideum) racF1, AAD09143; Ddiscoideum (Dictyostelium discoideum) rac 1A, P34144; Sbovinus (Suillus bovinus) rac, AF235004; Ylipolytica (Yarrowia lipolytica) rac, AF176831; Celegans (Caenorhabditis elegans) rac2, Q94124; Celegans (Caenorhabditis elegans) rac1, Q03206; Dmelanogaster (Drosophila melanogaster) rac1, P40792; Ggallus (Gallus gallus) rac, U79756; Xlaevis (Xenopus laevis) rac, AF174644; Hsapiens (Homo sapiens) rac1, P15154. from mycorrhizal short roots collected at different developmental stages (Niini et al. 1996). A clear signal of Cdc42 protein was obtained from early-stage ectomycorrhiza (stage 1) with an undivided short root that included either a forming or complete fungal sheath (Fig. 6A and C, lane E1) and from dichotomously branching mycorrhiza (stage 2) with two sheathed root tips (Fig. 6A and C, lane E2). Either a weak signal or no signal of Cdc42p was revealed by immunoblotting in extracts from coralloid stage mycorrhiza (stage 3), with three or more sheathed tips (Fig. 6C, lane E3). No signal was obtained with the $\mathrm{Cdc} 42$ antibody in the extracts from $P$.

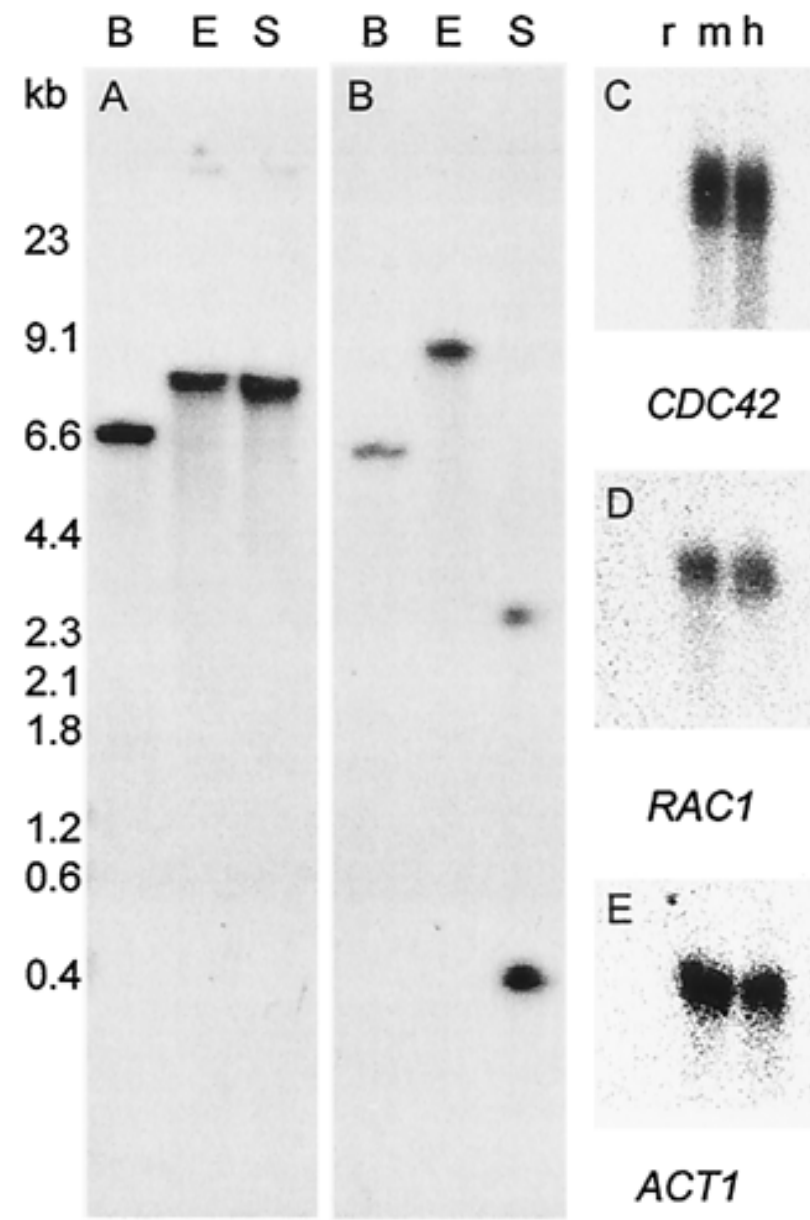

Fig. 5. A and B, Southern hybridization and $\mathbf{C}$ to $\mathbf{E}$, Northern hybridization of $S b C D C 42$ and $S b R A C 1$ genes. Hybridization of $\mathbf{B}$, BamHI-, $\mathbf{E}$, EcoRI-, and SalI (S)-endonuclease-digested Suillus bovinus genomic DNA with $S b C D C 42$ probe showed A, a single fragment and hybridization with $S b R A C l$ probe showed a single fragment in BamHI and EcoRI and $\mathbf{B}$, two fragments in SalI digested genomic DNA. SbRAC1 has an internal Sal restriction site. Hybridization of total RNA from Pinus sylvestris root cells (lane r), S. bovinus ectomycorrhiza (lane m), and $S$. bovinus hyphae (lane $\mathrm{h}$ ) with specific probes for C, $S b C D C 42$, D, $S b R A C 1$, and $\mathbf{E}, S b A C T 1$ genes show no hybridization with the RNA isolated from plant roots (lane r), but a hybridization signal for all genes in ectomycorrhiza and vegetative hyphae (lanes $\mathrm{m}$ and $\mathrm{h}$ ). A four-timesstronger signal was measured for $\mathbf{C}, S b C D C 42$ than occurred for the $\mathbf{D}$, $S b R A C 1$ gene when the expression was normalized by hybridizing the same filters with E, constitutively expressed SbACTl. No significant difference was observed in the expression rates of the $S b C D C 42$ or $S b R A C l$ genes between mycorrhizal and vegetative hyphae. Twenty micrograms of total RNA was loaded per lane, and equal loading was certified by ethidium bromide staining. 
sylvestris roots (Fig. 6C, lane P). The results were reproducible and obtained in three different experiments in which extracts from different hyphal and ectomycorrhizal samples were prepared for immunoblotting. Probing the extracts from $S$. bovinus hyphae, ectomycorrhizas, yeast cells (Fig. 6B), and $P$. sylvestris roots (not shown) with a monoclonal actin antibody revealed a clear band at $43 \mathrm{kDa}$, which is expected for actin. The $S$. cerevisiae Cdc42 antibody did not cross react with SbRaclp, as confirmed by comparing bacterially expressed SbCdc42p and SbRac1p (Fig. 6D and E).

No signal was obtained when protein extracts from $S$. bovinus vegetative or ectomycorrhizal hyphae and from $P$. sylvestris short roots were immunoblotted with a commercially available monoclonal antibody that detects human and plant Rac proteins.

\section{Localization of actin and Cdc42p in vegetative and ectomycorrhizal hyphae.}

Staining of $S$. bovinus vegetative hyphae with rhodaminelabeled phalloidin specific for filamentous actin (Fig. 7A to C) and the IIF-microscopic investigation with an anti-actin antibody (Fig. 7D and E) revealed the pattern of actin distribution known from previous immunocytochemical studies of $S$. bovinus and other homobasidiomycetes (Salo et. al. 1989; Timonen et al. 1993). Actin accumulated at hyphal tips (Fig. 7A, B, and D) and showed occasionally filamentous structures when stained with rhodamine-phalloidin (Fig. 7B). Microfilaments such as those in the short branch (Fig. 7B), however, are rarely detected in filamentous fungi. Some of the actin signal occurred as individual patches in the subapical part of the hypha (Fig. 7D) and as an actin ring at the site of the developing septum (Fig. 7C and E). The IIF microscopy of the polyclonal antibody against $\mathrm{Cdc} 42 \mathrm{p}$ indicated that $\mathrm{Cdc} 42 \mathrm{p}$ was, as actin, highly concentrated at the tip region of $S$. bovinus vegetative hyphae (Fig. $7 \mathrm{~F}$ ). Below the tip region, the signal obtained with the Cdc42 antibody became more diffuse and, in a few hyphae, stringlike structures were seen. At an average distance for septum formation, 40 to $50 \mu \mathrm{m}$ from the hyphal tip, the Cdc42 antibody visualized two opposite spots at plasma membrane in some hyphae (Fig. 7G). At the same distance from the hyphal tip, the actin ring preceding the formation of the septum was visualized regularly (Fig. 7C and E). In control hyphae where the polyclonal antibody against Cdc42p was omitted and the hyphae were labeled only with the rhodamine-conjugated secondary antibody, no strong signal was seen at the hyphal tips and weak fluorescence of rhodamine occurred only in the hyphae (Fig. 7H and I), which could cause some of the fluorescence seen in the subapical part of the hyphae labeled with the antibody against Cdc42p (Fig. 7F).

The majority of $S$. bovinus ectomycorrhizal hyphae separated from the root surface of the early-stage ectomycorrhiza, the same early stage that was sampled for Western blotting experiments (Fig. 6A to C, lane E1), had a swollen-tip region. Rhodamine-phalloidin staining showed that in the swollen-tip regions, the actin signal was less concentrated at the actual tip (Fig.7J, L, and M) than in the vegetative hyphae and could be apically depolarized (Fig. 7J). Instead, actin patches often were spread along the plasma membrane of the swollen-tip region (Fig. 7M). The actin ring, typical to the site of the septum formation in vegetative hyphae (Fig. 7C and E), was detected regularly in the ectomycorrhizal hyphae (Fig. 7K). When ectomycorrhizal root sections were labeled with the $\mathrm{Cdc} 42$ antibody, a strong signal for $\mathrm{Cdc} 42$ protein was detected below the plasma membrane of the swollen hyphal compartments (Fig. $7 \mathrm{O}$ and Q), typical to the ectomycorrhizal hyphae (Fig. 7P). When the Cdc42 antibody was omitted and the sections labeled only with the rhodamine-labeled secondary antibody, no localized fluorescence was observed (Fig. $7 \mathrm{R}$ ) in the hyphae that formed the loose sheath on the root surface (Fig. 7S).

\section{DISCUSSION}

We isolated from the ectomycorrhiza-forming filamentous fungus $S$. bovinus a $C D C 42$ and $R A C 1$ cDNA, both of which

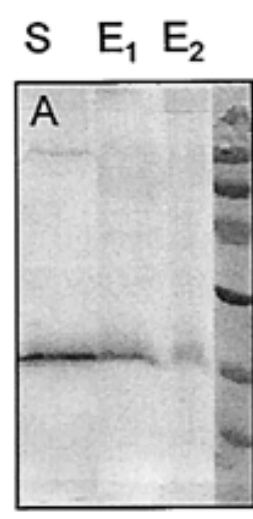

Anti-Cdc42
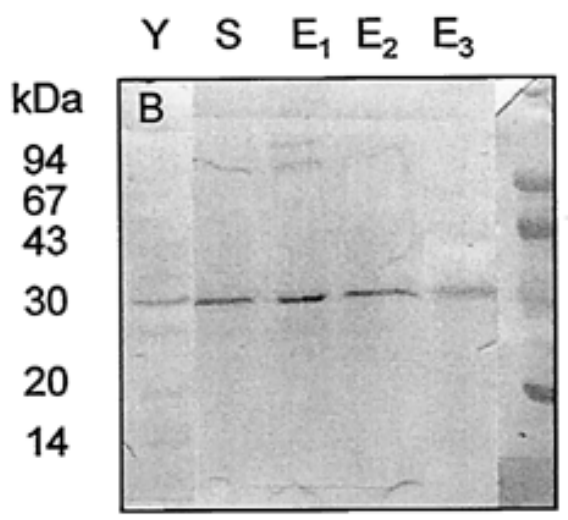

Anti-Actin

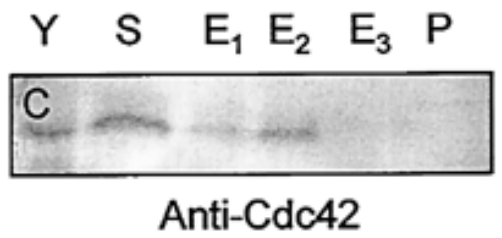

Cdc42 Rac Cdc42 Rac

30

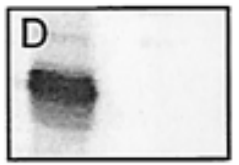

Anti-Cdc42

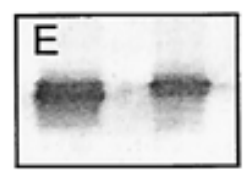

Anti-V5

Fig. 6. A and C, Immunoblot analysis of SbCdc42p and B, actin and D and E, SbCdc42p and SbRac1p expressed in Escherichia coli. A and C, Cdc42 antibody identified a single 21-kDa band from protein extracts of vegetative hyphae (lane S), ectomycorrhizae (lanes E1, early; E2, dichotomously branched; and E3, coralloid ectomycorrhiza), and yeast (lane Y). C, The Cdc42 signal was extremely low in coralloid ectomycorrhiza (lane E3) and the antibody did not react with plant protein extracts (lane P). B, Monoclonal anti-actin antibody detected a single band at $43 \mathrm{kDa}$, consisting of fungal and plant actins. D, Polyclonal antibody against yeast Cdc42p bound SbCdc42p, but not SbRac1p. E, SbCdc42p and SbRac1p were fused to V5 epitope in the bacterial expression vector. Successful heterologous expression in $E$. coli was confirmed by detection of V5 epitope in both proteins by immunoblotting with anti-V5 antibody. Fifteen micrograms of total protein was loaded per lane in $\mathbf{A}$ to $\mathbf{C}$, and $3 \mu \mathrm{g}$ of total protein was loaded per lane in $\mathbf{D}$ and $\mathbf{E}$. 
are single-copy genes as deduced from analysis of a large number of PCR clones and Southern hybridization. SbCDC42 and $S b R A C l$ are expressed in vegetative and symbiotic hyphae. For the $C D C 42$ gene, expression was further confirmed at the protein level by Western blotting and IIF microscopy.

$S b C D C 42$ is highly expressed in $S$. bovinus vegetative hyphae and ectomycorrhiza, as are the $S$. bovinus actin genes SbACT1 and SbACT2 (Tarkka et al. 2000). In previous studies, two-dimensional immunoblots of actin from vegetative hyphae and ectomycorrhizal roots at different developmental stages revealed an actin signal that was high in young vegetative hyphae and early mycorrhiza but decreased in coralloid mycorrhiza (Niini et al. 1996). A pattern comparable to that obtained with the actin antibody was detected in the immunoblots with the antibody against Cdc42p. A signal as strong as that for $\mathrm{Cdc} 42 \mathrm{p}$ in the vegetative hyphae was detected in young and dichotomously branched mycorrhiza, but it no longer was detected in samples from coralloid mycorrhiza, where the hyphal and root morphogenesis probably slowed or terminated. Different from the actin signal, which resulted from the interaction of fungal and plant actin with the antibody (Niini et al. 1996), the Cdc42p signal originated only from fungal hyphae because no signal from $P$. sylvestris short roots with the antibody against $\mathrm{Cdc} 42 \mathrm{p}$ was obtained. These results show that $\mathrm{Cdc} 42 \mathrm{p}$ and actin, the putative tools for regulation of polar growth of vegetative hyphae and hyphal morphogenesis in mycorrhiza, are expressed in the fungus as long as growth and differentiation of the hyphae take place.

Here $\mathrm{Cdc} 42 \mathrm{p}$ was immunodetected at the hyphal tip in the same region as actin, which shows that in filamentous fungi, Cdc42p is localized at the site of polarized growth, as it is in yeasts. A signal for $\mathrm{Cdc} 42 \mathrm{p}$ also was detected below the tip region. In animal cells the guanine nucleotide dissociation inhibitor (GDI) can sequester Cdc42p from the plasma membrane by interacting with switch domains I and II of Cdc42p and by inserting the $\mathrm{C}$-terminally attached geranylgeranyl moiety of Cdc42p in a hydrophobic pocket (Hoffman et al. 2000). In S. bovinus, the formation of the septum is associated with nuclear division and preceded by formation of an actin ring (Raudaskoski et al. 1991; Runeberg et al. 1986; Salo et al. 1989). In the hyphae immunostained with the antibody against Cdc42p, two small spots were found in some hyphae at the plasma membrane at the site that could be predicted to be the place of septal formation as deduced from its distance from the hyphal apex. This observation is of interest because in S. pombe, Cdc42p (Johnson 1999) and Cdc12p have been implicated in the actin ring formation necessary for cytokinesis (Chang et al. 1997). S. pombe Cdc12p is a homolog of the $S$. cerevisiae Cdc42-interacting protein Bnilp (Evangelista et al. 1997). In human and Xenopus spp. cells (Prokopenko et al. 2000), Cdc42 proteins are also part of a signal transduction pathway, mediating cytokinesis.

S. bovinus hyphae separated from ectomycorrhizal roots at the early stage of symbiosis had tips with different degrees of swelling, which suggests that the formation of swollen hyphal compartments originates from an apical-isotropic growth switch comparable to that known at bud development in the yeast S. cerevisiae (Johnson 1999), where the polarized anisotropic growth at the bud tip changes to isotropic growth after the bud has reached a certain size. This idea was further supported by the apically depolarized actin location in the hyphae, where the swelling had just started, and by the presence of actin and Cdc42p below the plasma membrane in the swollen compartments. In $S$. cerevisiae, the Cdc42p together with other proteins is known to regulate the organization of the actin cytoskeleton during polarized tip growth (Evangelista et al. 1997) as well as during isotropic enlargement of the bud (Johnson 1999), although the localization of $\mathrm{Cdc} 42 \mathrm{p}$ seems independent of the actin cytoskeleton (Ayscough et al. 1997). Several proteins associated with secretion at bud formation, however, require an intact actin cytoskeleton to reach the exocytosis site (Ayscough et al. 1997). In ectomycorrhiza during hyphal swelling, Cdc42p could direct the localization of the actin cytoskeleton, leading to the exceptional morphology of the symbiotic hyphae. The occurrence of branches in the swollen compartments indicates that polarized filamentous growth continues from the swollen compartments, perhaps by accumulation of Cdc $42 p$ and actin at the site of new growth by a mechanism not yet understood.

Here we show that $S$. bovinus has a $R A C$ gene, which is closely related to $C D C 42$. In addition to animals, several $R A C$ genes have been cloned from the cellular slime mold $D$. discoideum, A. thaliana, garden pea (Yang and Watson 1993), and rice (Kawasaki et al. 1999). Recently, a RAC gene also was cloned from the yeast $Y$. lipolytica (Hurtado et al. 2000), although the budding and fission yeasts have no $R A C$ genes. In animal cells, Rac proteins have a bifunctional role: they participate in actin organization (Albertinazzi et al. 1999; Nobes and Hall 1995) and act as obligatory participants in the superoxide generating NADPH oxidase (Toporik et al. 1998). In $D$. discoideum and plants, the bifunctional role of Rac proteins also occurs (Noegel and Schleicher 2000). In rice, Rac proteins are involved in the regulation of programmed cell death and the production of reactive oxygen species (ROS), probably through the NADPH oxidase (Kawasaki et al. 1999). In pollen tubes, Rac proteins are localized at the apex, where they are involved in maintaining a polarized growth pattern (Kost et al. 1999; Zheng and Yang 2000). The insert region specific to the Rho-family proteins is in human Rac proteins participating in NADPH oxidase activation (Freeman et al. 1996). Only low similarities between the "insert loop" amino acid sequences of human, plant, or SbRaclp exist and these cannot be used for function prediction. The participation of SbRac1p in the production of ROS and/or the cytoskeletal regulation remains to be clarified.

\section{MATERIALS AND METHODS}

\section{Biological material and growth conditions.}

For cDNA library construction, $S$. bovinus mycelia were grown on a $1.2 \%$ MMN agar medium (Molina and Palmer 1982), on which only half of the strength of organic nutrients was used, and malt extract was replaced with $2.5 \mathrm{~g}$ of glucose per liter. Two-week-old mycelia, $P$. sylvestris seedlings, and $P$. sylvestris ectomycorrhizal cultures were grown and harvested, as described previously (Niini et al. 1996; Tarkka et al. 1998). $S$. cerevisiae PLY15 wt (donated by P. Lappalainen) was grown at $30^{\circ} \mathrm{C}$ in liquid yeast extract-peptone-dextrose growth medium. 

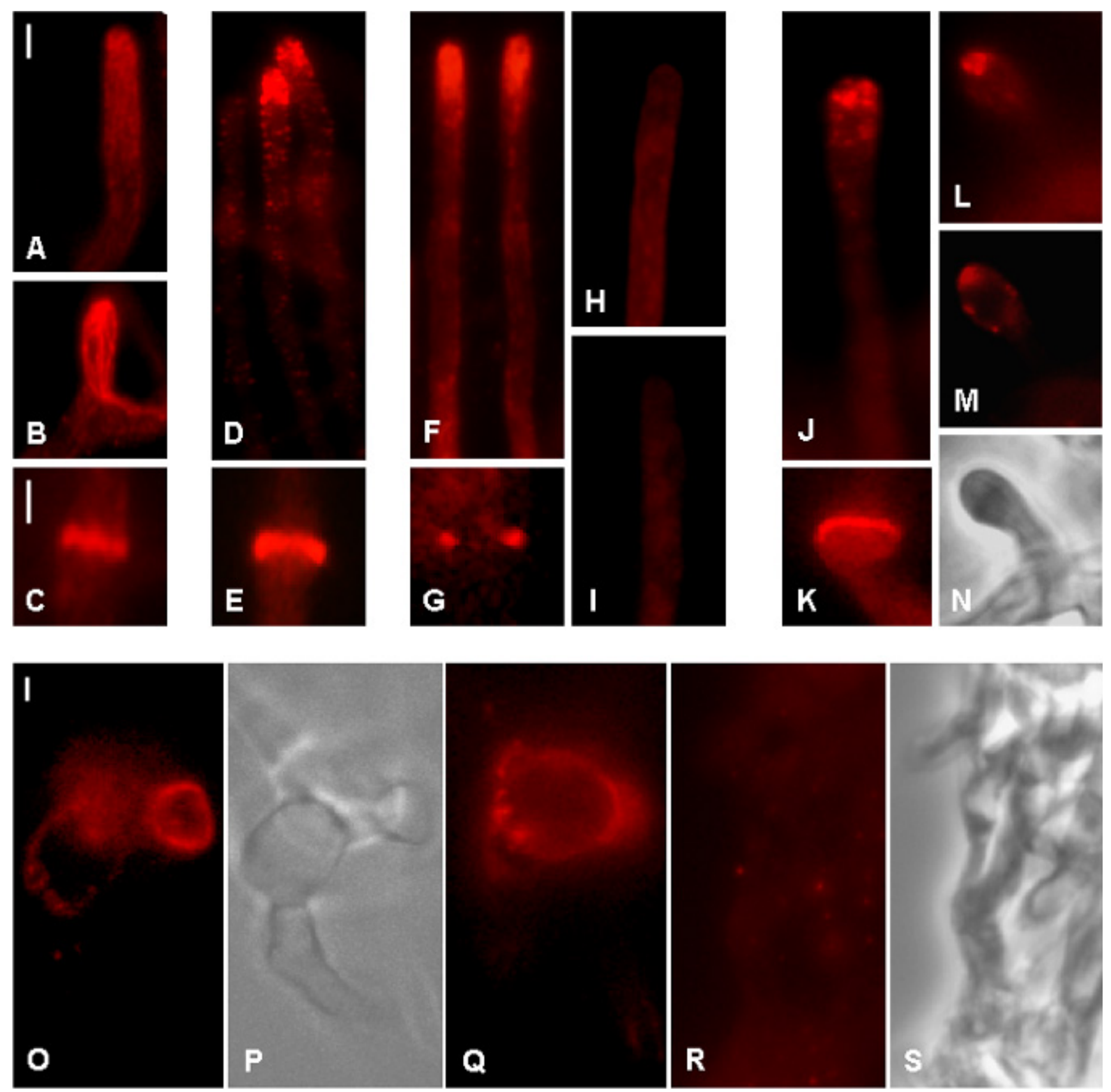

Fig. 7. A to $\mathbf{E}$ and $\mathbf{J}$ to $\mathbf{M}$, Localization of actin and $\mathbf{F}$ to $\mathbf{G}, \mathbf{O}$, and $\mathbf{Q}, \mathbf{C d c} 42 p$ in $\mathbf{A}$ to $\mathbf{I}$, Suillus bovinus vegetative and $\mathbf{J}$ to $\mathbf{S}$, ectomycorrhizal hyphae. A, Rhodamine-phalloidin staining of actin at the tip of a leading hypha, $\mathbf{B}$, a short branch, and $\mathbf{C}$, actin ring at the site of septum formation. D, Indirect immunofluorescence microscopy with monoclonal anti-actin antibody visualizes actin at the hyphal tips and $\mathbf{E}$, at the actin ring at the site of septum formation, similar to rhodamine-phalloidin staining. F, Indirect immunofluorescence microscopy with the polyclonal antibody against Cdc42p leads to strong fluorescence at the hyphal tips and diffuse fluorescence along the subapical part of the hyphae. G, Two spots with strong fluorescence are detected opposite to each other at the site of septum formation. H to I, In control hyphae labeled only with the rhodamine-conjugated secondary antibody, no fluorescence at the hyphal tips is seen and weak fluorescence occurs only in the subapical part of the hyphae. J, Rhodamine-phalloidin staining of apically depolarized actin patches in the swollen-tip region and $\mathbf{K}$, at the actin ring in ectomycorrhizal hyphae. $\mathbf{L}$ and $\mathbf{M}$, Rhodamine-phalloidin staining and $\mathbf{N}$, phase-contrast microscopy of an ectomycorrhizal hypha with swollen-tip region. $\mathbf{L}$ and $\mathbf{M}$ represent different focal planes of the same hyphae. $\mathbf{L}$, Actin patches are seen at the hyphal tip and $\mathbf{M}$, along the plasma membrane below the tip. $\mathbf{O}$ and $\mathbf{Q}$, Indirect immunofluorescence microscopy with Cdc42 antibody and $\mathbf{P}$, phase-contrast microscopy of a section cut from young ectomycorrhiza. $\mathbf{O}$ and $\mathbf{Q}$, The Cdc $42 p$ occurs in association with plasma membrane of swollen hyphal cells. The diffuse fluorescence in $\mathbf{O}$ above the swollen hyphal parts originates from a third swollen compartment shown in focus in $\mathbf{Q}$. Note the branch originating from the swollen cell in $\mathbf{P}$. R, Control section of hyphal mantle from young ectomycorrhiza. Without the Cdc42 antibody, only weak, nonspecific fluorescence is seen in the sections of ectomycorrhizal hyphae shown with phase-contrast microscopy in $\mathbf{S}$. Bars $=10 \mu \mathrm{m}$. 
DNA and RNA isolations and construction

of an $S$. bovinus cDNA library.

Genomic DNA was extracted from $S$. bovinus with the DNeasy Plant Mini Kit (Qiagen, Hilden, Germany). Total RNA was isolated from $S$. bovinus, $P$. sylvestris nonmycorrhizal short roots, and mycorrhizae, as described in Nehls et al. (1998).

For construction of the $S$. bovinus cDNA-library, $50 \mu \mathrm{g}$ of total RNA were reverse transcribed with Superscript II (GIBCO BRL, Eggenstein, Germany) and cloned into the $\lambda$ ZAP vector, according to the manufacturer's instructions (Stratagene, Amsterdam, The Netherlands).

\section{Primers and PCR conditions.}

For cDNA library screening, $S b C D C 42$ and $S b R A C 1$ gene fragments were amplified from genomic DNA with primers C1 (5'-AARTGYGTNGTNGTNGGNGAC-3') and C4 (5'CAYTCNACRTAYTTNACNGC-3') for $S b C D C 42$ and primers R1 (5'-GTNGGNGAYGGNGCNGTNGGNAARAC-3') and R2 (5'-TARTCYTCYTGNCCNGCNGTRTC-3') for SbRAC1. The PCR program for both amplifications was initial denaturation at $95^{\circ} \mathrm{C}$ for $2 \mathrm{~min}, 30$ cycles of $94^{\circ} \mathrm{C}$ for $45 \mathrm{~s}$, $58^{\circ} \mathrm{C}$ for $45 \mathrm{~s}, 72^{\circ} \mathrm{C}$ for $45 \mathrm{~s}$, and a final extension at $72^{\circ} \mathrm{C}$ for $5 \mathrm{~min}$. For expression in Escherichia coli, a truncated SbCDC42-cDNA from pSbCdc42 was amplified with primers SbCdc42-1 (5'-CAGACTATCAAGGTTGTAGT-3') and SbCdc42-2 (5'-TTTATGTGTCTTCTTAACCAC-3') and initial denaturation at $95^{\circ} \mathrm{C}$ for $2 \mathrm{~min}, 20$ cycles of $94^{\circ} \mathrm{C}$ for $45 \mathrm{~s}$, $56^{\circ} \mathrm{C}$ for $45 \mathrm{~s}, 72^{\circ} \mathrm{C}$ for $1 \mathrm{~min}$, and a final extension at $72^{\circ} \mathrm{C}$ for $5 \mathrm{~min}$. A truncated $S b R A C l \mathrm{cDNA}$ from pSbrac1 was amplified with primers Sbrac1-1 (5'-CACAACATCAAATGTGT TGTA-3') and Sbrac1-3 (5'-ACCACGACCACTGCGCTT-3') and initial denaturation at $95^{\circ} \mathrm{C}$ for $2 \mathrm{~min}, 20$ cycles of $94^{\circ} \mathrm{C}$ for $45 \mathrm{~s}, 52^{\circ} \mathrm{C}$ for $45 \mathrm{~s}, 72^{\circ} \mathrm{C}$ for $1 \mathrm{~min}$, and a final extension at $72^{\circ} \mathrm{C}$ for $5 \mathrm{~min}$.

Routinely, $10 \mathrm{ng}$ of genomic DNA or $0.1 \mathrm{ng}$ of plasmid DNA were used as a template, $\mathrm{MgCl}_{2}$ concentration was 1.5 $\mathrm{mM}$, DyNAzyme Polymerase (Finnzymes, Espoo, Finland) was used as the thermostable enzyme in the buffer supplied by the manufacturer, and obtained products were subcloned into pCR2.1 (Invitrogen, Leek, The Netherlands). PCR products for expression in $E$. coli were inserted in pBAD-TOPO (Invitrogen). The cDNAs and PCR products were sequenced automatically with the ABI Prism TM 377 DNA Sequencer (Perkin-Elmer, Foster City, CA, U.S.A.) and samples were prepared with the BigDye TM Terminator Cycle Sequencing Ready Reaction Kit (Perkin-Elmer).

\section{Hybridization procedures.}

Nylon membranes were used for library screenings, and all hybridization steps were carried out under stringent conditions. Southern blot analysis was performed as described in Russo et al. (1992). For Northern blot analyses, $20 \mu \mathrm{g}$ of total RNA was denatured at $55^{\circ} \mathrm{C}$ in the presence of formamide and formaldehyde, separated on $1.5 \%$ agarose gel in $20 \mathrm{mM}$ morpholinepropanesulfonic acid buffer, and transferred to nylon membranes. DNA-fragments were labeled to high specific activity with $\alpha-{ }^{32} \mathrm{P}-\mathrm{dCTP}$ and Random Labeling Kit $1600 \mathrm{Z}$ (Amersham-Pharmacia Biotech, Buckinghamshire, U.K.). Relative expression levels of different genes were approximated by phospho-imager analysis with the BAS-1500 Bio- imaging Analyzer (Fuji, Düsseldorf, Germany) and Tina 2.0 software (Raytest Isotopengeräte, Straubenhardt, Germany). SbCdc42 and SbRac1 expression levels were normalized to constitutively expressed SbACT1 (Tarkka et al. 2000).

\section{Protein extraction, polyacrylamide gel electrophoresis (PAGE), and immunoblotting.}

The transfer of proteins from the unstained gels to nitrocellulose filters and the immunodetection of $\mathrm{Cdc} 42$ by a polyclonal antibody against $S$. cerevisiae Cdc42p (donated by D. Kellogg, University of California, Santa Cruz, U.S.A.) and of actin by monoclonal anti-actin antibody MAB 1501 (Chemicon, Temecula, CA, U.S.A.) were performed as described by Niini et al. (1996). For expression of S. bovinus Cdc42 and Rac1 in E. coli TOP10 (Invitrogen), protein production was induced by $0.2 \%$ arabinose. Bacterial proteins were isolated by boiling the cells in $1 \times$ sodium dodecyl sulfate (SDS)-PAGE sample buffer. After centrifugation, total proteins were separated by SDS-PAGE and Western blotted with the same anti-Cdc42 antibody and a commercial anti-V5 antibody (Invitrogen).

\section{Indirect immunofluorescence microscopy and related methods.}

For immunocytochemical staining of S. bovinus vegetative hyphae, the methods developed previously for localization of cytoskeletal elements in filamentous fungi were used (Raudaskoski et al. 1991; Raudaskoski et al. 1994; Rupeš et al. 1995; Torralba et al. 1998). Small mycelial colonies of $S$. bovinus were grown on dialysis membranes covered with a thin layer of $0.5 \%$ agarose and overlying nutrient medium for 4 to 5 days. The colonies still attached to the membranes were quick frozen and freeze substituted, as described previously (Raudaskoski et al. 1991). After freeze substitution, the samples were gradually rehydrated and immunolabeled with the same antibodies as used for immunodetection of actin and Cdc42p by Western blotting.

For IIF microscopic localization of $\mathrm{Cdc} 42 \mathrm{p}$ in ectomycorrhizal hyphae, the procedure developed by Baluska et al. (1995 and 1997) for immunodetection of cytoskeletal elements in plant roots was used, with some modifications. As in previous investigations on cytoskeleton of ectomycorrhizal pine roots (Niini and Raudaskoski 1998; Timonen et al. 1993), intact $P$. sylvestris seedlings with ectomycorrhizal short roots were prefixed for $1 \mathrm{~h}$ in a vacuum with $4 \%$ formaldehyde in $50 \mathrm{mM}$ piperazine- $N, N^{\prime}$, bis(2-ethanesulfonic acid) (PIPES) buffer, containing $5 \mathrm{mM} \mathrm{MgSO} 4,5 \mathrm{mM}$ ethylene glycol-bis( $\beta$ aminoethyl ether) $N, N, N^{\prime}, N^{\prime}$-tetraacetic acid, and $\mathrm{pH}$ set to 6.9 with $\mathrm{KOH}$. After prefixation, the young ectomycorrhizal short roots were removed from the root system under the fixative, and fixation was continued for 2 to $3 \mathrm{~h}$. The samples were rinsed with the same PIPES buffer as used in fixation and dehydrated in a graded ethanol series diluted with phosphatebuffered saline (PBS), $\mathrm{pH}$ 7.0. The wax used for embedding was prepared by melting and mixing $900 \mathrm{~g}$ of poly(ethyleneglycol-400)-distearate (Aldrich, Milwaukee, WI, U.S.A.) and $100 \mathrm{~g}$ of 1-hexadecanol (cetyl alcohol) (ICN Pharmaceuticals Inc., Costa Mesa, CA, U.S.A.) for 3 to $5 \mathrm{~h}$ at $60^{\circ} \mathrm{C}$. Wax infiltration was performed at $37^{\circ} \mathrm{C}$ in a graded wax-ethanol series, followed by three changes in pure wax at $37^{\circ} \mathrm{C}$ in a vacuum, with each step lasting at least $2 \mathrm{~h}$. After infiltration, 
the roots were placed in embedding molds, covered with wax, and left to polymerize at room temperature overnight. Sections 5 to $10 \mu \mathrm{m}$ thick were collected on SuperFrost plus slides (Menzel-Gläser, Braunschweig, Germany) and allowed to dry overnight. Wax was removed from the sections by treating them with $100 \%$ acetone for $10 \mathrm{~min}$, after which they were rinsed several times with PBS, $\mathrm{pH}$ 7.3.

For immunolocalization, sections were first kept in blocking solution containing $0.1 \%$ bovine serum albumin (BSA) and $0.1 \%$ Triton $\mathrm{X}-100$ in PBS, pH 7.3, for 20 min and then incubated with the monoclonal anti-actin or polyclonal antiCdc42 antibodies at a 1:250 dilution in PBS overnight. After several rinses with PBS containing $0.1 \%$ BSA, the sections treated with the anti-actin antibody were incubated with rhodamine-conjugated goat anti-mouse secondary antibody, whereas those treated with polyclonal antibody against Cdc42p were incubated with rhodamine-conjugated goat antirabbit secondary antibody (Jackson Immuno Research Laboratories, West Grove, PA, U.S.A.). Both incubations occurred overnight at a dilution of 1:40. The specific interaction of the polyclonal antibody against Cdc $42 p$ was tested by omitting the primary antibody from the IIF microscopic procedure of the quick-frozen and freeze-substituted vegetative hyphae and mycorrhizal root sections.

For staining of filamentous actin with rhodamine-labeled phalloidin (Molecular Probes, Leiden, The Netherlands), mycelial colonies on dialysis membranes and $P$. sylvestris seedlings with ectomycorrhizal short roots were fixed for $1 \mathrm{~h}$ in vacuum with $4 \%$ formaldehyde in PIPES buffer, $\mathrm{pH}$ 6.9. After fixation, the young ectomycorrhizal short roots were cut from the root system in PIPES buffer. Mycelial colonies and short roots were rinsed several times in the PIPES buffer, after which the material was transferred to the rhodamine phalloidin staining solution modified from Traas et al. (1987). The staining solution was prepared in the same PIPES buffer as used for fixation and washes by adding $0.33 \mu \mathrm{M}$ of rhodamine-labeled phalloidin, $0.1 \%$ Triton $\mathrm{X}-100,3 \mathrm{mM}$ dithiothreitol, $50 \mu \mathrm{g}$ of leupeptine per $\mathrm{ml}, 20 \mu \mathrm{g}$ of aprotinin per $\mathrm{ml}$, and $1 \mathrm{mM}$ phenylmethylsulfonyl fluoride. The ectomycorrhiza-forming hyphae were drawn out carefully from the short roots in the staining solution. The ectomycorrhizal and vegetative hyphae were then spread in the staining solution as well as possible for microscopic examination.

All the samples were viewed with a DM LB research microscope (Leica, Wetzlar, Germany) equipped with 4-lambda Reflected Light Fluorescence Illuminator and a $100 \mathrm{~W}$ mercury lamp (Leica). Images were captured electronically with a PCO CCD 12 BIT Cooled Imaging Video Camera (SensiCam, Kelheim, Germany) and displayed on an OptiPlexGxiP computer (Dell Computer Corp., Round Rock, TX, U.S.A.) with Image-Pro Plus software (Media Cybernetics, Silver Spring, MD, U.S.A.).

\section{ACKNOWLEDGMENTS}

This work was supported by grants from the Academy of Finland to M. Raudaskoski and from the Universidad Nacional de Quilmes, Argentina and Center for International Mobility, Finland, to A. G. Pardo.

\section{LITERATURE CITED}

Albertinazzi, C., Cattelino, A., and de Curtis, I. 1999. Rac GTPases localize at sites of actin reorganization during dynamic remodeling of the cytoskeleton of normal embryonic fibroblasts. J. Cell Sci. 112:3821-3831.

Ayscough, K. R., Stryker, J., Pokala, N., Sanders, M., Crews, P., and Drubin, D. G. 1997. High rates of actin filament turnover in budding yeast and roles for actin in establishment and maintenance of cell polarity revealed using the actin inhibitor latrunculin-A. J. Cell Biol. 137:399-416.

Baluska, F., Barlow, P. W., Hauskrecht, M., Kubica, Š., Parker, J. S., and Volkmann, D. 1995. Microtubule arrays in maize root cells. Interplay between the cytoskeleton, nuclear organization and post-mitotic cellular growth patterns. New Phytol. 130:177-192.

Baluska, F., Vitha, S., Barlow, P. W., and Volkmann, D. 1997. Rearrangements of F-actin arrays in growing cells of intact maize root apex tissues: A major developmental switch occurs in the postmitotic transition region. Eur. J. Cell Biol. 72:113-121.

Bölker, M. 1998. Sex and crime: Heterotrimeric G proteins in fungal mating and pathogenesis. Fungal Genet. Biol. 25:143-156.

Chang, F., Drubin, D., and Nurse, P. 1997. Cdc12p, a protein required for cytokinesis in fission yeast, is a component of the cell division ring and interacts with profilin. J. Cell Biol. 137:169-182.

Couchy, I., Minic, Z., Laporte, J., Brown, S., and Satiat-Jeunemaitre, B. 1998. Immunodetection of Rho-like plant proteins with Rac1 and Cdc42Hs antibodies. J. Exp. Bot. 49:1647-1659.

Evangelista, M., Blundell, K., Longtine, M. S., Chow, C. J., Adams, N., Pringle, J. R., Peter, M., and Boone, C. 1997. Bnilp, a yeast formin linking $\mathrm{Cdc} 42 \mathrm{p}$ and the actin cytoskeleton during polarized morphogenesis. Science 276:118-122.

Felsenstein, J. 1989. PHYLIP-phylogeny interference package. Cladistics 5:164-166.

Freeman, J. L., Abo, A., and Lambeth, J. D. 1996. Rac "insert region" is a novel effector region that is implicated in the activation of NADPH oxidase, but not PAK65. J. Biol. Chem. 271:19794-19801.

Hicks, J. K., Yu, J. H., Keller, N. P., and Adams, T. H. 1997. Aspergillus sporulation and mycotoxin production both require inactivation of the FadA G alpha protein-dependent signaling pathway. EMBO J. 16:4916-4923.

Hoffman, G. R., Nassar, N., and Cerione, R. A. 2000. Structure of the Rho family GTP-binding protein Cdc42 in complex with the multifunctional regulator Rho GDI. Cell 100:345-356.

Hurtado, C. A., Beckerich, J. M., Gaillardin, C., and Rachubinski, R. A. 2000. A rac homolog is required for induction of hyphal growth in the dimorphic yeast Yarrowia lipolytica. J. Bacteriol. 182:2376-2386.

Johnson, D. I. 1999. Cdc42: An essential Rho-type GTPase controlling eukaryotic cell polarity. Microbiol. Mol. Biol. Rev. 63:54-105.

Johnson, D. I., and Pringle, J. R. 1990. Molecular characterization of CDC42, a Saccharomyces cerevisiae gene involved in the development of cell polarity. J. Cell. Biol. 111:143-152.

Kawasaki, T., Henmi, K., Ono, E., Hatakeyama, S., Iwano, M., Satoh, H., and Shimamoto, K. 1999. The small GTP-binding protein rac is a regulator of cell death in plants. Proc. Natl. Acad. Sci. USA 96:1092210926.

Kost, B., Lemichez, E., Spielhofer, P., Hong, Y., Tolias, K., Carpenter, C., and Chua, N.-H. 1999. Rac homologues and compartmentalized phosphatidylinositol 4,5-bisphosphate act in a common pathway to regulate polar pollen tube growth. J. Cell Biol. 145:317-330.

Kottke, I., and Oberwinkler, F. 1986. Mycorrhiza of forest trees-structure and function. Trees 1:1-24.

Molina, R., and Palmer, J. G. 1982. Isolation, maintenance, and pure culture manipulation of ectomycorrhizal fungi. Pages 115-129 in: Methods and Principles of Mycorrhizal Research. N. C. Schenck, ed. American Phytopathological Society, St. Paul, MN, U.S.A..

Nehls, U., Wiese, J., Guttenberger, M., and Hampp, R. 1998. Carbon allocation in ectomycorrhizas: Identification and expression analysis of an Amanita muscaria monosaccharide transporter. Mol. Plant-Microbe Interact. 11:167-176.

Niini, S. S., and Raudaskoski, M. 1998. Growth patterns in nonmycorrhizal and mycorrhizal short roots of Pinus sylvestris. Symbiosis 25:101-114.

Niini, S. S., Tarkka, M. T., and Raudaskoski, M. 1996. Tubulin and actin protein patterns in Scots pine (Pinus sylvestris) roots and developing ectomycorrhiza with Suillus bovinus. Physiol. Plant. 96:186-192.

Nobes, C. D., and Hall, A. 1995. Rho, rac, and cdc42 GTPases regulate the assembly of multimolecular focal complexes associated with actin stress fibers, lamellipodia, and filopodia. Cell 81:53-62. 
Noegel, A. A., and Schleicher, M. 2000. The actin cytoskeleton of Dictyostelium: A story told by mutants. J. Cell Sci. 113:759-766.

Nuoffer, C., and Balch, W. E. 1994. GTPases: Multifunctional molecular switches regulating vesicular traffic. Annu. Rev. Biochem. 63:949-990.

Omer, C. A., and Gibbs, J. B. 1994. Protein prenylation in eukaryotic microorganisms: Genetics, biology and biochemistry. Mol. Microbiol. 11:219-225.

Prokopenko, S. N., Saint, R., and Bellen, H. J. 2000. Untying the Gordian knot of cytokinesis: Role of small $\mathrm{G}$ proteins and their regulators. J. Cell Biol. 148:853-848.

Raudaskoski, M., Mao, W.-Z., and Yli-Mattila, T. 1994. Microtubule cytoskeleton in hyphal growth. Response to nocodazole in a sensitive and a tolerant strain of the homobasidiomycete Schizophyllum commune. Eur. J. Cell Biol. 64:131-141.

Raudaskoski, M., Rupeš, I., and Timonen, S. 1991. Immunofluorescence microscopy of the cytoskeleton in filamentous fungi after quickfreezing and low-temperature fixation. Exp. Mycol. 15:167-173.

Rosen, S., Yu, J. H., and Adams, T. H. 1999. The Aspergillus nidulans $s f a D$ gene encodes a $\mathrm{G}$ protein beta subunit that is required for normal growth and repression of sporulation. EMBO J. 18:5592-5600.

Runeberg, P., Raudaskoski, M., and Virtanen, I. 1986. Cytoskeletal elements in the hyphae of the homobasidiomycete Schizophyllum commune visualized with indirect immunofluorescence and NBDphallacidin. Eur. J. Cell. Biol. 41:25-32.

Rupeš, I., Mao, W.-Z., Åström, H., and Raudaskoski, M. 1995. Effects of nocodazole and brefeldin A on microtubule cytoskeleton and membrane organization in the homobasidiomycete Schizophyllum commune. Protoplasma 185:212-221.

Russo, P., Juuti, J. T., and Raudaskoski, M. 1992. Cloning, sequence and expression of a $\beta$-tubulin-encoding gene in the homobasidiomycete Schizophyllum commune. Gene 119:175-182.

Salo, V., Niini, S., Virtanen, I., and Raudaskoski, M. 1989. Comparative immunocytochemistry of the cytoskeleton in filamentous fungi with dikaryotic and multinucleate hyphae. J. Cell. Sci. 94:11-24.

Schmidt, A., and Hall, M. N. 1998. Signalling to the actin cytoskeleton. Annu. Rev. Cell Dev. Biol. 14:305-338.

Tarkka, M., Niini, S. S., and Raudaskoski, M. 1998. Developmentally regulated proteins during differentiation of root system and ectomycorrhiza in Scots pine (Pinus sylvestris) with Suillus bovinus. Physiol. Plant 104:449-455.

Tarkka, M. T., Vasara, R., Gorfer, M., and Raudaskoski, M. 2000. Molecular characterization of actin genes from homobasidiomycetes: Two different actin genes from Schizophyllum commune and Suillus bovinus. Gene 251:27-35.

Timonen, S., Finlay, R. D., Söderström, B., and Raudaskoski, M. 1993. Identification of cytoskeletal components in pine ectomycorrhizas. New Phytol. 124:83-92.

Toporik, A., Gorzalczany, Y., Hirshberg, M., Pick, E., and Lotan, O. 1998. Mutational analysis of novel effector domains in Rac1 involved in the activation of nicotinamide adenine dinucleotide phosphate (reduced) oxidase. Biochemistry 37:7147-7156.

Torralba, S., Raudaskoski, M., Pedregosa, A. M., and Laborda, F. 1998. Effect of cytochalasin A on apical growth, actin cytoskeleton organization and enzyme secretion in Aspergillus nidulans. Microbiology 144:45-53.

Traas, J. A., Doonan, J. H., Rawlins, D. J., Shaw, P. J., Watts, J., and Lloyd, C. W. 1987. An actin network is present in the cytoplasm throughout the cell cycle of carrot cells and associates with the dividing nucleus. J. Cell Biol. 105:387-395.

Yang, Z., and Watson, J. C. 1993. Molecular cloning and characterization of Rho, a Ras-related small GTP-binding protein from the garden pea. Proc. Natl. Acad. Sci. USA 90:8732-8736.

Zheng, Z.-L., and Yang, Z. 2000. The Rop GTPase switch turns on polar growth in pollen. Trends Plant Sci. 5:298-303. 